\title{
Laparoscopic transabdominal cervical cerclage: Case report of a woman without exocervix at 11 weeks gestation
}

\author{
Jae Eun Shin, Min Jeong Kim, Guk Won Kim, Dae Woo Lee, Min Kyung Lee, Sa Jin Kim \\ Department of Obstetrics and Gynecology, The Catholic University of Korea College of Medicine, Seoul, Korea
}

Cervical incompetence is characterized by painless dilatation of the incompetent cervix and results in miscarriages and preterm delivery during second trimester. We report a 25-year-old patient, gravid 2, para 1, at 11 weeks' gestation with the diagnosis of cervical incompetence, in whom transvaginal cerclage was not technically possible and laparoscopic cervical cerclage was performed successfully. There were no operative or immediate postoperative complications. A healthy infant was delivered at 35 weeks by cesarean section. Laparoscopic cervical cerclage during pregnancy can be safe and effective treatment for well-selected patients with cervical incompetence and eliminates the need for open laparotomy.

Keywords: Cervical cerclage; Laparoscopy; Pregnancy

\section{Introduction}

Cervical incompetence occurs in $0.5 \%$ to $1 \%$ of all pregnancies and has a recurrence risk of $30 \%$. Patients typically present pelvic pressure and cervical dilatation in the absence of uterine activity or ruptured membranes in the first and second trimester [1].

Therapeutic or prophylactic cervical cerclage is recommended treatment for cervical incompetence. It is typically performed transvaginally during the first trimester for those patients with a confirmed diagnosis of cervical incompetence who are pursuing normal childbearing. However, in a small subset of patients, the vaginal approach is not feasible because of anatomic cervical distortions, either congenital or as a result of previous surgical procedures. For these women, the transabdominal route has been successfully used. Laparoscopic surgical techniques have now increasingly replaced traditional abdominal approaches to gynecologic surgery.

This article describes our experience with the laparoscopic placement of cervical cerclage in a woman who had severe cervical deformities and very short cervix without exocervix due to extensive loop electrosurgical excision procedure (LEEP).

\section{Case report}

A 25-year-old woman at 9 weeks of gestation, gravid 2, para 1, was referred to our hospital with a highly suggestive of cervical incompetence. She had a past history of preterm delivery at 26 weeks before. After delivery she received two times of LEEP because of microinvasive squamous cell carcinoma of the cervix.

On physical examination, the cervix was exceedingly short, closed, and flush with the lateral vaginal fonix. Transvaginal ultrasound scanning confirmed a singleton intrauterine pregnancy and the size of fetus was consistent with 9 weeks of

Received: 2013.3.13. Revised: 2013.10.3. Accepted: 2013.10.31. Corresponding author: Sa Jin Kim

Department of Obstetrics and Gynecology, Bucheon St. Mary's Hospital, The Catholic University of Korea, 327 Sosa-ro 327beongil, Wonmi-gu, Bucheon 420-717, Korea Tel: +82-32-340-2262 Fax: +82-32-340-2663

E-mail: ksajin@catholic.ac.kr

Articles published in Obstet Gynecol Sci are open-access, distributed under the terms of the Creative Commons Attribution Non-Commercial License (http://creativecommons. org/licenses/by-nc/3.0/) which permits unrestricted non-commercial use, distribution, and reproduction in any medium, provided the original work is properly cited.

Copyright $\odot 2014$ Korean Society of Obstetrics and Gynecology 


\section{Obstetrics \& Gynecology Science}

Jae Eun Shin, et al. Laparoscopic abdominal cerclage

gestation.

Her cervical length was short with a functional length of 1.70 $\mathrm{cm}$. It was determined that she was a candidate for a transabdominal cerclage because of virtually nonexistent exocervix. Extensive counseling regarding the risks and benefits of the procedure performed either laparoscopically or via laparotomy were provided. The patient was taken to surgery at 11 weeks for laparoscopic cervical cerclage.

Under general anesthesia, the patient was placed in the dorsal lithotomy position, and a Foley catheter was inserted. No vaginal instrumentation was performed. Peritoneal access was established by laparoscopy. An intraabdominal pressure of $12 \mathrm{mmHg}$ was maintained during procedure. The patient was placed in steep trendelenburg position. A 10-mm port was introduced through an umbilical incision. One secondary 5-mm trocar was placed through the suprapubic area and two 5-mm trocars were placed in each lower quadrant laterally.

The vesicouterine peritoneum was dissected transversely with the laparoscopic scissors and the bladder was carefully reflected downward to expose the uterine isthmus and uterine vessels. The transverse dissection was generous enough to provide an anterior visualization of the course of the uterine arteries.

The suture was done by the 5 -mm mersilene tape and the needle was straightened before insertion. After uterine vessels were identified, the needle was introduced medial and posterior to the uterine vessels at the level of the cervicoisthmic junction. And the needle was followed through right anteriorly with the distal end of the suture in the cul-de-sac behind the uterus. The suture material and needle was passed posteriorly across the lower segment to the left side of the uterus. In a similar fashion, the needle was placed medial to the left sided uterine vessels at the level of the left sided cervicoisthmic junction. The ligature was pulled snugly against the posterior cervical isthmus, and the ligature ends were tied together anteriorly by intracorporeal knot tying (Fig. 1). Reteritonealization was not carried out. Perioperative antibiotics were administered. The operation lasted 137 minutes and was successful. The estimated blood loss was $150 \mathrm{~mL}$. Fetal cardiac activity was confirmed before and after the procedure. The patient was discharged from the hospital after a 24-hour observation period. No tocolytic agents were used at the time or after the procedure. After the operation, postoperative findings were checked that mersilene tape was placed in proper position in the cervix by the transvaginal 2- and 3-dimensional ultraso-

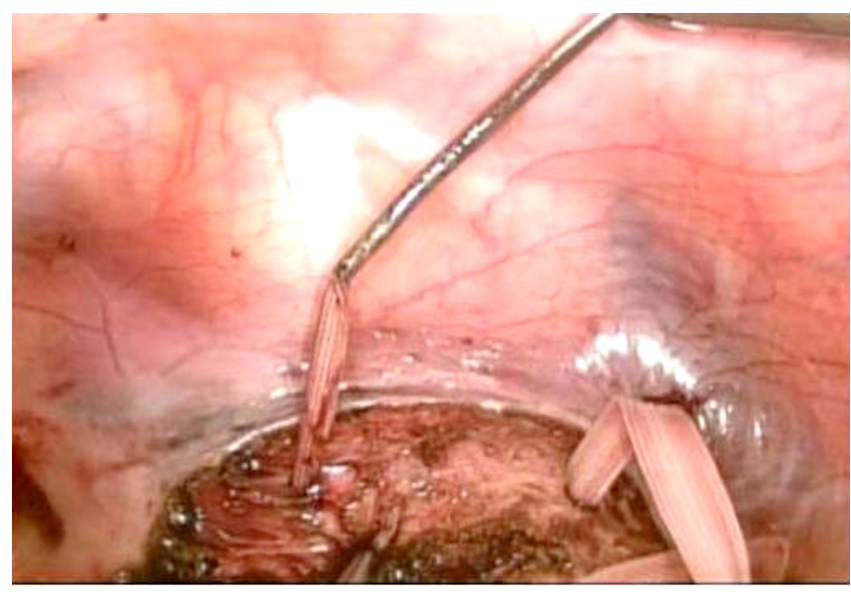

Fig. 1. The 5-mm mersilene tape that was tied anteriorly with an intracorporeal surgeon's knot.

\section{nography (Fig. 2).}

The rest of her pregnancy was unremarkable. She was scheduled to undergo a planned cesarean section for 37 weeks of gestation, but the patient went into labor at 35 weeks of gestation. She underwent at low transverse cesarean delivery and the ligature was removed. The infant weighted 2,460 $\mathrm{g}$ with Apgar scores of 7 and 8. The mother and the baby did well after surgery.

\section{Discussion}

Cervical incompetence has been traditionally treated with a cerclage placed in the vaginal portion of the cervix in the subsequent pregnancy [1]. The treatment of choice for the last 50 years was cervical cerclage [2].

The vaginal approach was developed in 1955 and was associated with a significant failure rate in cases of anatomical distortion in the cervix [3]. Large LEEP of transformation zone procedures to the cervix are now increasing. The cervical suture can be difficult to place in the cervix that is shortened or scarred from previous laser or LEEP conization. Suture placement at the internal cervical os is imprecise, and leaves a foreign body and potential site for infection in the vagina.

Transabdominal cervicoisthmic cerclage was first performed in 1965 as an alternative to transvaginal cervical cerclage [4]. The transabdominal approach is beneficial in women with short cervices of congenital origin or secondary to previous surgical procedures, and in those with severely lacerated cervices due to obstetric trauma [5]. However, its main disadvan- 


\section{Obstetrics \& Gynecology Science}

Vol. 57, No. 3, 2014
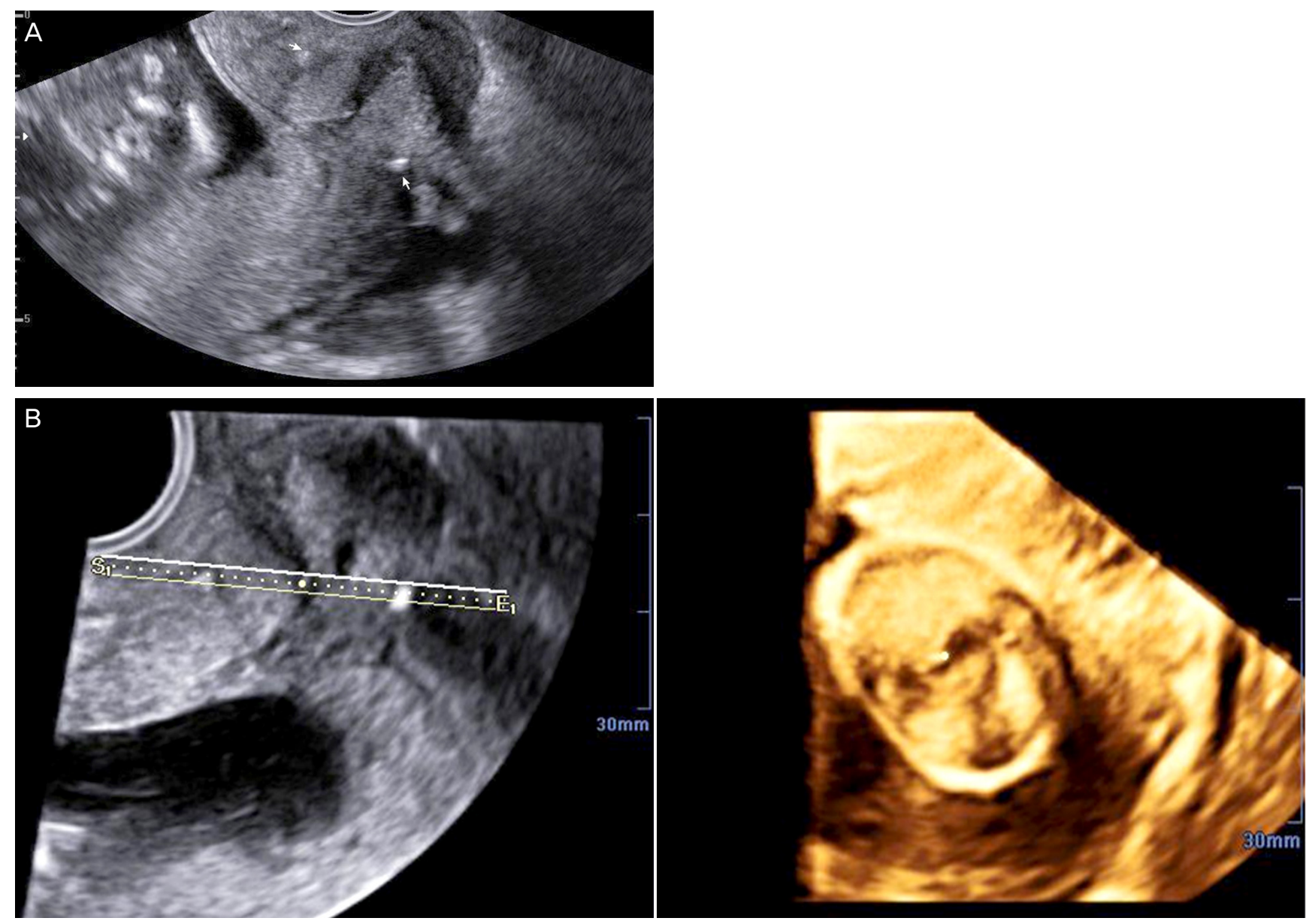

Fig. 2. Postoperative ultrasound images of the cervix. (A) The cervix with mersilene tape (arrow). (B) 3D image of Mersine tape in the cervix.

tage is the need for two open laparotomies, one for cerclage and one for cesarean section. Postoperative bowel adhesion is also associated with the open method and requires longer hospitalization and recovery [5].

Laparoscopic surgical techniques have now increasingly replaced traditional abdominal approaches. Laparoscopic placement of cervical cerclage has many theoretic advantages. Laparoscopy provides superior views of the uterine cervicoisthmic junction compared with vaginal or abdominal surgery. This allows close approximation to the level of the internal cervical os when placing the suture laparoscopically. The procedure can be safely performed before pregnancy, avoiding the need for surgery during pregnancy [6]. Complications of conventional cervical cerclage include chorioamnionitis, and this may be a result of the presence of the suture in the cervix [2]. Laparoscopic cerclage also offers the benefits of reduced hospital stay and a faster recovery. According to one of the largest reported case series of 20 patients who underwent transabdominal cerclage, the hospital stay ranged from 4 to 7 days [7]. Previous studies reported the usage of a gauze ball or instruments vaginally for the manipulation of the uterus [5], but there were no vaginal approach performed in this case for avoiding cervical injury or bleeding. Instead, we used one more $5 \mathrm{~mm}$-trochar for handling the uterus to assist for suture.

This case support the conclusion that laparoscopic cervical cerclage is a minimally invasive, extremely safe and effective procedure in properly selected patients and should replace the traditional laparotomy technique. Minor modifications of the previously reported techniques may be helpful in reducing the risks of the procedure.

\section{Conflict of interest}

No potential conflict of interest relevant to this article was reported. 


\section{Obstetrics \& Gynecology Science}

Jae Eun Shin, et al. Laparoscopic abdominal cerclage

\section{References}

1. Shennan A, Jones B. The cervix and prematurity: aetiology, prediction and prevention. Semin Fetal Neonatal Med 2004;9:471-9.

2. Harger JH. Cerclage and cervical insufficiency: an evidence-based analysis. Obstet Gynecol 2002;100:1313-27.

3. Schwartz RP, Chatwani A, Sullivan P. Cervical cerclage: a review of 74 cases. J Reprod Med 1984;29:103-6.

4. Benson RC, Durfee RB. Transabdominal cervico uterine cerclage during pregnancy for the treatment of cervical incompetency. Obstet Gynecol 1965;25:145-55.

5. Cho CH, Kim TH, Kwon SH, Kim JI, Yoon SD, Cha SD. Laparoscopic transabdominal cervicoisthmic cerclage during pregnancy. J Am Assoc Gynecol Laparosc 2003;10:363-6.

6. Liddell HS, Lo C. Laparoscopic cervical cerclage: a series in women with a history of second trimester miscarriage. J Minim Invasive Gynecol 2008;15:342-5.

7. Novy MJ. Transabdominal cervicoisthmic cerclage: a reappraisal 25 years after its introduction. Am J Obstet Gynecol 1991;164(6 Pt 1):1635-41. 